IMECE2021-68722

\title{
SIZING OPTIMIZATION OF DISTRICT ENERGY SYSTEMS CONSIDERING METEOROLOGICAL, DEMAND, AND ELECTRICITY EMISSION FACTOR UNCERTAINTIES
}

\author{
Zahra Ghaemi \\ Dept. of Mechanical Engineering \\ University of Utah \\ Salt Lake City, UT 84112 \\ Email: zahra.ghaemi8@gmail.com
}

\author{
Thomas T. D. Tran \\ College of Engineering \\ Indiana Institute of Technology \\ Fort Wayne, IN 46803
}

\author{
Amanda D. Smith ${ }^{1,2}$ \\ ${ }^{1}$ Dept. of Mechanical Engineering \\ University of Utah \\ Salt Lake City, UT 84112 \\ 2 Pacific Northwest National Laboratory \\ Richland, WA, 99352
}

\begin{abstract}
In this study, a framework is developed to perform two-stage stochastic programming in a district energy system. This framework optimizes the sizing of energy components to minimize the total cost and operating $\mathrm{CO}_{2}$ emissions. Uncertainties in electricity demand, solar irradiance, wind speed, and electricity emissions are considered. A group of buildings at University of Utah is used as the case study to test the optimization framework. This study is novel by forming an open-source framework, considering electricity emissions with more details compared to previous studies in the literature, and performing the optimization for a campus in the U.S. This study's results show the trade-off between cost and emissions when different energy configurations are used for three electricity purchasing cases. This framework can help facility managers to evaluate the optimum sizing of their district energy system to minimize the cost and emissions.
\end{abstract}

\section{NOMENCLATURE}

$C A P_{i} \quad$ A vector that holds the maximum capacity of energy components $(\mathrm{kW})$

DES District energy system

EGEF Electricity generation emission factor

EIA Energy Information Administration

$I C_{i} \quad$ A vector that holds the investment cost per capacity $(\$ / \mathrm{kW})$ of energy components (i)
LS Lifespan of the DES (years)

NREL National Renewable Energy Laboratory

NSRDB National Solar Radiation Database

NSGA-II Non-dominated sorting genetic algorithm II O\&M Operation \& maintenance

$O P_{i, r, h} \quad$ Operating energy of the energy components (i) in each hour $(\mathrm{h})$ of the representative days $(\mathrm{r})(\mathrm{kWh})$

$O P C_{r, h} \quad$ Operating cost of the DES in each hour (h) of the representative days $(\mathrm{r})(\$)$

$O P E_{r, h} \quad$ Operating emissions of the DES in each hour (h) of the representative days (r) $(\mathrm{kg} \mathrm{CO})$

$O \& M$ var $_{i} \quad$ Variable O\&M cost of energy components $(\$ / \mathrm{kWh})$

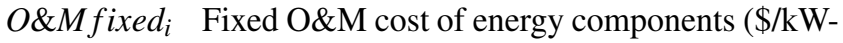
y)

PV Photovoltaics

Prob $_{r} \quad$ A vector that holds the normalized probability of occurrence of each representative day (r), where the sum of the vector is one

SP Stochastic programming

U.S. United States

\section{INTRODUCTION}

The most significant driver of climate change globally is the increase in greenhouse gas emissions caused by human activities 
( [1] and [2]). The increase in greenhouse gas emissions has catastrophic effects on human health, the ecosystem, and society [3]. As a result, countries, cities, and campuses have developed emission mitigation policies to reduce emissions caused by human activities.

One of the emission reduction plans by the United Nations Environment Programme is using a district energy system (DES) connected to renewables [4]. DESs can reduce emissions compared to purchasing power from the grid by using renewable energy sources and reducing the waste energy during transmission and distribution in the grid [5].

DESs are versatile in their applications and ability to connect different renewables sources. DESs can provide heating, cooling, and/or electricity needs for buildings, neighborhoods, and campuses. They can be connected to different energy components to generate heating and/or electricity in a centralized location and distribute them to buildings. A DES can be connected to a solar photovoltaic (PV) system or a wind turbine for electricity generation. Due to the intermittent nature of electricity generation from renewable sources, energy storage systems also can be utilized in DESs to store excess generated electricity from renewables.

The objective of DESs can be the minimization of cost and emissions, maximization of resiliency, providing backup power, and/or reaching $100 \%$ renewables goals. Cost is a critical optimization objective of DESs as it justifies the profitability and feasibility of a project [6]. Emissions are also an essential optimization objective due to campuses' and cities' present emission mitigation goals. Given the various DES objectives, decisionmakers can use multi-objective optimization to study the trade-off between the total cost and emissions by generating a Pareto-front.

Many studies neglected the changes in solar irradiance, wind speed, electricity demands of buildings, and electricity emissions over the lifespan of DESs. However, the electricity consumption of buildings may change in the future. For instance, the U.S. Energy Information Administration (EIA) predicted that the cooling and electricity demands of commercial buildings would reduce by $31 \%$ and $10 \%$ from 2021 to 2050 in the U.S. [7]. Also, electricity emissions from the grid may change over the years. For example, University of Utah developed a climate action plan to reduce its emissions and be carbon neutral by 2050 [8]. This plan aims to purchase electricity from more renewable sources over the years. This plan can change the campus emissions associated with purchasing electricity from the grid in the future. Therefore, weather intermittency, changes in electricity, and changes in electricity emissions can happen over the lifespan of DESs and should be considered in the design of DESs.

Stochastic programming (SP) is an approach to optimize problems that involve uncertainties [9]. Two-stage SP has been used to optimize the design of DESs under uncertainties [10]. Two-stage SP splits decision variables into two groups. The first group of decision variables are related to the design of DESs and are made before realizing uncertainties, which means uncer- tainties do not affect the design parameters. Stage one decision variables are the sizing of energy components in a DES. Once the uncertainties are realized, further operational adjustments can be made through stage two decision variables [11]. Stage two decision variables in a DES are the operation planning of energy components. These two stages of decision variables should be optimized to result in optimum designs/sizings of the DES.

Two research groups have performed a multi-objective twostage SP of sizing of energy components (e.g., solar PV, wind turbines, and batteries) in DESs connected to renewables to minimize the cost and emissions considering uncertainties. Mavromatidis et al. ( [10], [12], [13], and [14]) used the CPLEX solver to optimize design of DESs in Switzerland under a wide range of variable inputs. Wang et al. ( [15], [16], and [17]) used the Gurobi solver to optimize the design of DESs in China under uncertain building energy demands, energy carrier prices, and solar radiation. There are three gaps in stochastic optimization of the sizing of energy components sizing in DESs connected to renewables under uncertainties by these two research groups:

1. Emissions uncertainties are neglected by Wang et al., and emission uncertainties are considered using a uniform probability distribution by Mavromatidis et al.

2. An open-source framework that can help future researchers modify the characteristics (e.g., capacities, efficiencies, investment cost, operation $\&$ maintenance $(\mathrm{O} \& \mathrm{M})$ cost $)$ of energy components or test a different case study is not available.

3. The location of case studies that performed stochastic multiobjective optimization of DESs are limited to China and Switzerland.

In this study, we develop a framework to perform a two-stage SP for DESs. This framework optimizes the sizing of energy components to minimize the total cost (investment, O\&M, and operating cost) and operating $\mathrm{CO}_{2}$ emissions. Uncertainties in electricity demand, solar irradiance, wind speed, and electricity $\mathrm{CO}_{2}$ emissions are considered in the framework. This study is novel by forming an open-source framework, considering electricity $\mathrm{CO}_{2}$ emissions with more details compared to Mavromatidis et al. and Wang et al. studies, and performing the optimization for a DES in the U.S.

\section{METHOD}

In this method section, system description, uncertainty analysis, mathematical statements, and two-stage SP are covered.

\section{System Description}

The proposed DES is shown in Figure 1. Four buildings at the University of Utah are selected to represent the demand side of the DES. Three buildings are class-office space, and one building 


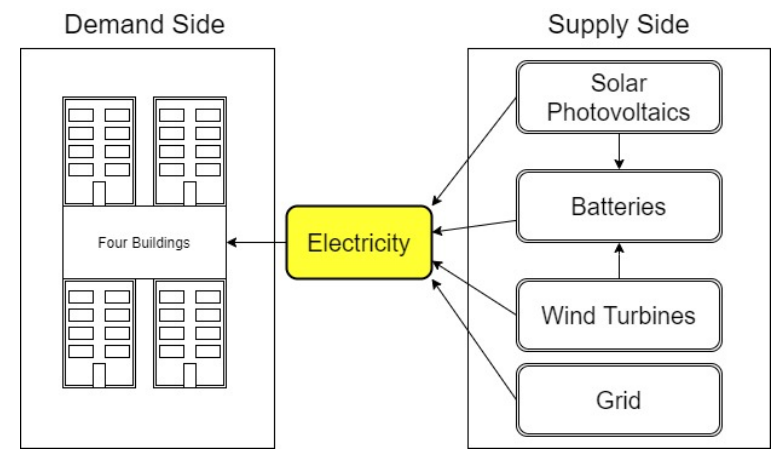

FIGURE 1: Proposed district energy system

is a physical fitness space. The usable floor areas of the three buildings are between 50,000 to 75,000 $\mathrm{ft}^{2}$, and the usable floor area of one building is over 100,000 $\mathrm{ft}^{2}$.

Solar PV, wind turbines, and the grid supply the electricity demand of the DES. Electricity generation from the solar PV system and wind turbines is first used for the buildings' electricity demand. Batteries are utilized to store the excess electricity generated from the solar PV system and wind turbines when the buildings' electricity demands are lower than the electricity generation from the on-site renewable sources. The stored electricity in the batteries is used when the buildings' electricity demand is higher than the electricity generation from the on-site renewable sources.

\section{Uncertainty Analysis}

We used a similar process to perform uncertainty analysis as Mavromatidis et al. ( [10], [12], [13], and [14]) and Wang et al. ( [16]) to consider the design of DESs under uncertainties. Compared to Mavromatidis et al. and Wang et al.'s studies, we have one additional step, "Filling the data gaps", as we used meter data that may include suspect and missing values. Also, we included the wind speed and electricity emissions in addition to electricity demand and solar irradiance in the representative days (the output of the scenario reduction step).

The uncertainty analysis consists of four steps in this study: (1) filling the data gaps, (2) scenario generation, (3) scenario reduction, and (4) preservation of important scenarios, which is shown with more details in Figure 2.

(1) Filling the data gaps: Electricity and cooling demands are from real buildings at the University of Utah. These meter data can contain suspect (i.e., negative or extremely high consumption values) or missing data. We followed a simplified processing method for meter data analysis [18] to identify the suspect and missing data and fill electricity and cooling demand gaps in this study. The data are processed to ensure there are no gaps greater than one week in the meter data. In this method, the data gaps smaller than twenty-four hours are estimated by linear interpo-

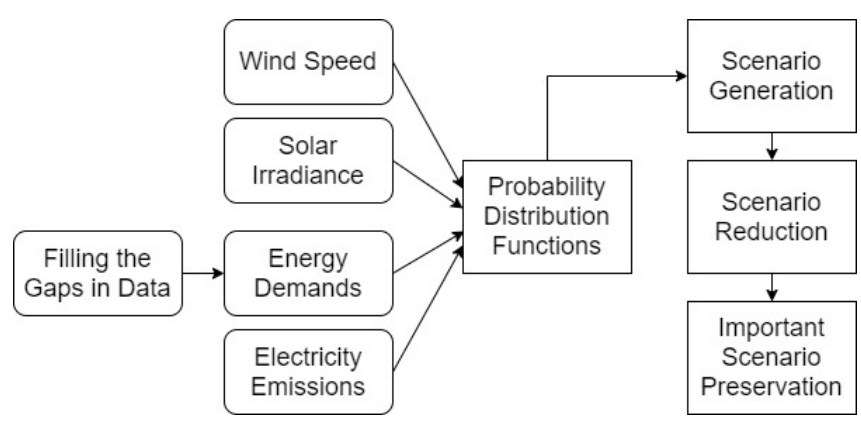

FIGURE 2: Uncertainty analysis of sizing optimization of district energy systems

lation of data over that day. The gaps greater than one day and smaller than one week are filled by linear interpolation of the equivalent day of week and time of the day before and after the gap.

(2) Scenario generation: Scenarios for (a) electricity demand, (b) solar irradiance and wind speed, and (c) electricity $\mathrm{CO}_{2}$ emissions are generated based on projections on their values over the lifespan of the DES.

(a) Electricity demand scenarios: EIA predicted that electricity and cooling demands per square feet of an individual commercial building decrease linearly from 2020 to 2050 by $9 \%$ and $31 \%$ [7]. Linear changes in the total electricity can be represented as a uniform distribution. As the total electricity decreases from 2020 to 2050 , this uniform distribution's maximum value is in 2020, and its minimum value is in 2050 .

Discrete approximation of continuous probability distribution function is used to select three points from different probability distribution functions in the literature ( [10], [16], and [19]). For discrete approximation of a standard uniform distribution, Miller et al. [19] suggested parameter values of $0.11,0.5$, and 0.88 with probabilities of $0.28,0.44$, and 0.28 . Hence, three points are generated for each hour of the base year to represent electricity demand scenarios.

(b) Solar irradiance and wind speed scenarios: Solar irradiance and wind speed data in Salt Lake City (University of Utah's location) from 1998 to 2019 are available from the National Solar Radiation Database (NSRDB) [20]. Salt Lake City's solar irradiance and wind speed data from NSRDB are used to form probability distribution functions for the base year. Therefore, 8760 (number of hours in a year) probability distribution functions are generated for solar irradiance and wind speed, separately. Similar to scenario generation for electricity, the discrete approximation of the continuous probability distribution function is used. Three points are generated for each hour in the base year for solar irradiance and wind speed, separately, using the discrete approximation of probability distribution functions ( [10], [16], and [19]).

(c) Electricity $\mathrm{CO}_{2}$ emissions scenarios: To consider the 
changes in electricity $\mathrm{CO}_{2}$ emissions over the years, quantification of emissions associated with electricity generation in the grid is necessary. An emission factor can be used to quantify the emissions related to electricity generation. An emission factor connects the quantity of an emitted pollutant with an activity related to the release of that pollutant [21]. An electricity generation emission factor (EGEF) calculates the emissions released per unit of electricity generation ( $\mathrm{kg}$ emissions/MWh generated).

We consider two types of uncertainties in the EGEF in this study:

First, uncertainties associated with $\mathrm{CO}_{2}$ EGEF values due to variability in fossil fuel sources' emissions to generate electricity are considered. An uncertainty analysis method is used from a previous paper submitted for publication by the authors [22] to consider uncertainties due to variability in emissions of fossil fuel sources to generate electricity. In this method, Utah's EGEF is quantified using a weighted sum of the fossil fuel emissions for Utah's fuel mix. Fossil fuel emissions vary due to changes in fossil fuel emissions across different regions, sectors, and types of combustion devices. The discrete approximation of the continuous probability distribution function is used to generate three points from the probability distribution function of EGEF for each hour of the base year ( [10], [16], and [19]).

Second, uncertainties are present due to the University of Utah's strategic plans to purchase more renewable sources from the grid over the years and go carbon neutral by 2050 [8]. Three cases of power purchasing are considered in this study to assess uncertainties in electricity $\mathrm{CO}_{2}$ emissions over the years at the University of Utah:

1. The University of Utah uses $100 \%$ grid electricity.

2. The University of Utah uses a mix of $29 \%$ grid electricity and $71 \%$ renewables. This case is considered because the University of Utah agreed to a new 25-year solar energy contract. It is expected to deliver power by mid-2022 [23], which results in purchasing $71 \%$ of the University of Utah's electricity from renewable sources.

3. The University of Utah goes carbon neutral, and $100 \%$ of its electricity is purchased from renewable sources.

(3) Scenario reduction: Electricity demand, solar irradiance, wind speed, and EGEF data are available in 2019 for the DES's location. Electricity demand data is from energy meters at the University of Utah, actual values of solar irradiance and wind speed data are from NSRDB [20], and EGEF values are from the U.S. EIA [24]. After scenario generation, eighty-one year-equivalents of synthetic data of electricity demand, solar irradiance, wind speed, and EGEF are generated. Eighty-one comes from $3^{4}=81$, as three data points from the discrete approximation of continuous probability distribution are generated for the four uncertain inputs (i.e., electricity demand, solar irradiance, wind speed, and EGEF) in the base year.

Eighty-one year-equivalents of synthetic data that contains
365 days and 24 hours of electricity demand, solar irradiance, wind speed, and EGEF are available. Using and optimizing this data is computationally expensive and almost infeasible. Therefore, a scenario reduction technique is necessary to reduce the number of scenarios and neglect similar scenarios.

As each day contains twenty-four hours of four parameters (i.e., electricity demand, solar irradiance, wind speed, and electricity $\mathrm{CO}_{2}$ emissions data), 96 features are needed to represent each day $(24 \times 4=96)$ in this study. With 96 features for each day, feature extraction is performed to characterize the variation of each parameter by using a selected number of features ( [10] and [16]).

Principal components analysis has been used as a feature extraction technique before scenario reduction techniques in the literature ( [25] and [26]). Therefore, we use principal components analysis and extract eighteen features to represent each day in the scenario reduction section. The explained variance that measures the fit of a regression [27] is used as a metric to choose the number of features. Eighteen features are selected to preserve $99.5 \%$ of the explained variance within the original data.

We choose the k-medoid clustering algorithm to reduce the number of scenarios in this study. K-medoid clustering algorithm has been used in the literature to reduce the number of scenarios in the design of DESs under uncertainties ( [10] and [15]). K-medoid algorithm partitions the scenarios into $\mathrm{k}$ groups or clusters so that scenarios within a cluster are similar to each other and dissimilar to scenarios in the other clusters [28]. The representative scenarioor in our study, the representative day-is the most centrally located day in each cluster [28].

The shortcoming of the k-medoid clustering algorithm is that the user must define the number of clusters. The Elbow method has been used to find the optimum number of clusters when the k-medoid clustering algorithm is used in the literature ( [29], [30], and [31]). The Elbow method uses inertia to indicate the performance of the clustering algorithm [32]. Inertia is the sum of distances of samples to their closest cluster center. Figure 3 shows the inertia versus the number of clusters. An elbow point, a point where a drastic change is in the inertia value, and there is no significant change in the inertia of the next point [33], is shown in Figure 3. Therefore, using the elbow method to find the optimum number of clusters, we use 9 clusters in the k-medoid algorithm.

(4) Preservation of extreme scenarios: After employing the k-medoid algorithm for nine clusters, nine representative days that are the most centrally located day in each cluster are selected. Each representative day consists of twenty-four hours of electricity demand, solar irradiance, wind speed, and EGEF. As these representative days are the most central day of each cluster, extreme electricity day is neglected by the k-medoid algorithm. One additional day to represent extreme electricity day is added, which results in ten representative days. We used the method described in [10] and [34] to generate the extreme electricity scenario. In 


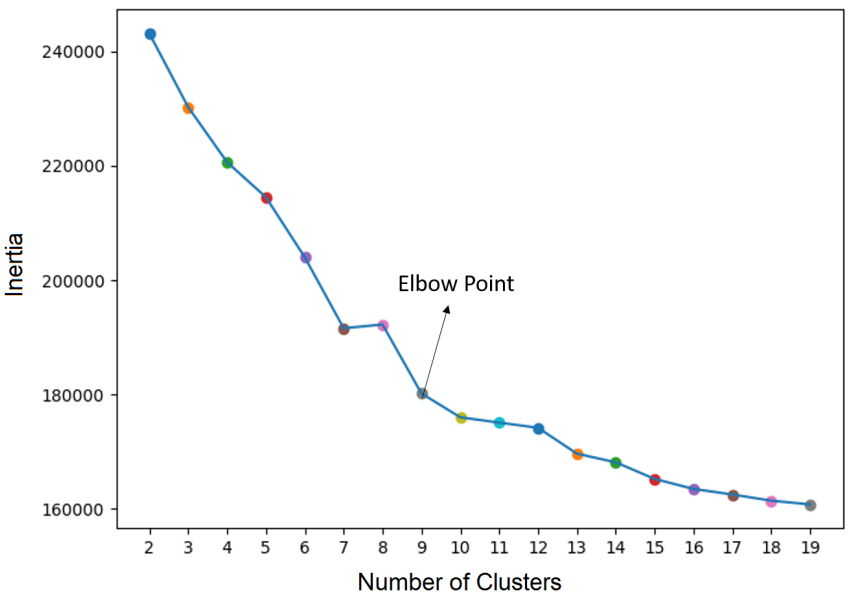

FIGURE 3: Inertia, the sum of distances of samples to their closest cluster center, verses the number of clusters. Elbow point is shown to find the optimum number of clusters in the k-medoid algorithm.

this method, electricity unmet hours are limited to 35 hours per year $(0.04 \%$ of the year) for $99 \%$ of the scenarios.

Each representative day is the most centrally located day in each cluster. The occurrence probability of each representative day depends on the number of days that belong to that cluster. Thus, each representative day is assigned a probability equal to the sum of probabilities of days in that cluster.

\section{Mathematical Statements}

The capital cost of the three energy components is quantified in Equation 1, where $C A P_{i}$ is a vector of length three (number of energy components) that holds the maximum capacity of energy components. $I C_{i}$ is a vector of length three that holds the investment cost per capacity $(\$ / \mathrm{kW})$ of energy components. $I C_{i}$ values are shown in Table 1. The lifespan of the DES is assumed as twenty years in this study. The lifespan is assumed as twenty years because solar PV and wind turbines' lifespans, shown in Table 1, are at least twenty years. Batteries' lifespan is ten years, and their substitution after ten years is considered in the capital cost to guarantee the DES operates efficiently for twenty years.

$$
\text { Capital Cost }=\sum_{i=1}^{3} C A P_{i} \times I C_{i}
$$

The total operating cost over the life span of a DES is quantified in Equation 2. Prob $r$ is a vector of length ten that holds the normalized occurrence probabilities of ten representative days, where the sum of the vector is one. Each representative day is
TABLE 1: Energy components characteristics to quantify their capital and operating cost

\begin{tabular}{ccccc}
\hline $\begin{array}{c}\text { Energy } \\
\text { components }\end{array}$ & $\begin{array}{c}\text { Investment cost per } \\
\text { capacity }(\$ / \mathrm{kW})\end{array}$ & $\begin{array}{c}\text { Fixed O\&M } \\
\text { cost }(\$ / \mathrm{kW} \text {-year) }\end{array}$ & $\begin{array}{c}\text { Variable O\&M } \\
\text { cost }(\$ / \mathrm{kWh})\end{array}$ & $\begin{array}{c}\text { Lifespan } \\
(\text { years })\end{array}$ \\
\hline Solar PV & $1830[35]$ & $18[35]$ & $0[36]$ & $30[37]$ \\
\hline Wind turbines & $1740[38]$ & $44[39]$ & $0[36]$ & $20[36]$ \\
\hline Battery & $2338[40]$ & $6[41]$ & $0[41]$ & $10[42]$ \\
\hline
\end{tabular}

the most central day of a cluster, where some days with similar characteristics belong to that cluster. The occurrence probability of each representative day is quantified by calculating the sum of probabilities of the days that belong to each cluster in the $\mathrm{k}$-medoid clustering algorithm.

$O P C_{r, h}$ is a matrix with ten rows (number of representative days) and twenty-four columns (twenty-four hours in each representative day) that holds the operating cost of the DES in each hour of the representative days.

$$
\text { Total Operating Cost }=\sum_{r=1}^{10} \operatorname{Prob}_{r} \times \sum_{h=1}^{24} O P C_{r, h}
$$

$O P C_{r, h}$ for each hour of the representative days is quantified in Equation 3 by getting the variable operating cost of the the grid. The variable cost of the solar PV, wind turbines, and batteries are assumed zero in this study ( [36] and [41]). $O P_{i, r, h}$ is a matrix that holds the hourly operation energy of batteries and the grid $(\mathrm{kWh})$ for each representative day. The operating energy for batteries is the stored energy in them, and is purchased electricity for the grid. Electricity price is the projected average rate of electricity from University of Utah utility rates and bill collection disbursement [43].

$$
O P C_{r, h}=\text { Electricity price } \times \mathrm{OP}_{g r i d, r, h}
$$

The operating cost of the solar PV, wind turbines, and batteries is quantified using their fixed O\&M cost per energy unit per year, their capacities, and the DES's lifespan, shown in Equation 4. O\&M fixed ${ }_{i}$ is a vector of length three that holds the fixed O\&M cost per energy unit per year $(\$ / \mathrm{kW}$-year) of the three energy components, shown in Table 1. LS is the lifespan of the DES, which is assumed as twenty years.

$$
\text { O\&M fixed cost }=\sum_{i=1}^{3} \text { O\&M fixed }_{i} \times \mathrm{CAP}_{i} \times \mathrm{LS}
$$

The first objective is minimizing the sum of capital cost (Equation 


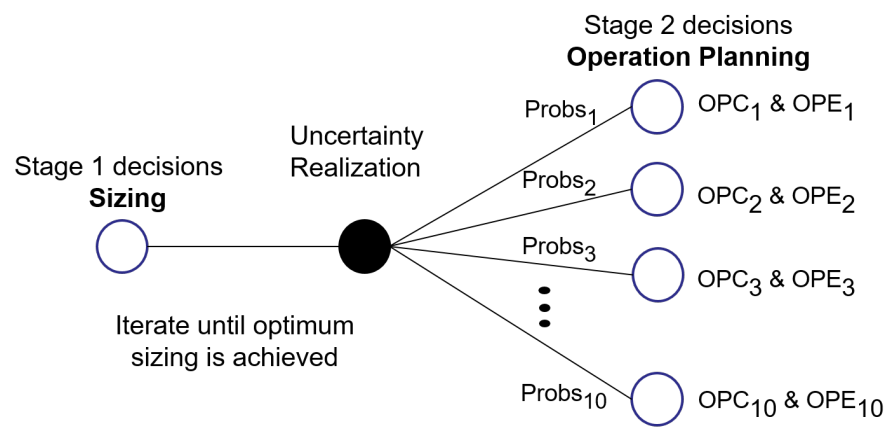

FIGURE 4: Two-stage stochastic optimization diagram

1), total operating cost over the life span of the DES (Equation 2), and fixed O\&M cost (Equation 4), which is shown in Equation 5.

$$
\begin{gathered}
\text { Min } f_{1}(X)=\operatorname{Min}(\text { Capital Cost }+ \text { Total Operating Cost }+ \\
\text { O\&M fixed cost })
\end{gathered}
$$

The second objective is minimizing the total operating $\mathrm{CO}_{2}$ emissions over the life span of the DES, which is shown in Equation 6. The embodied emissions of the DES are neglected due to current limitations in quantifying life cycle emissions of large scale systems [44].

$$
\begin{aligned}
\operatorname{Min} f_{2}(X) & =\operatorname{Min}(\text { Total Operating Emissions) } \\
& =\operatorname{Min} \sum_{r=1}^{10} \operatorname{Prob}_{r} \times \sum_{h=1}^{24} O P E_{r, h}
\end{aligned}
$$

$O P E_{r, h}$ is a matrix with ten rows (number of representative days) and twenty-four columns (twenty-four hours in each representative day) that holds the operating $\mathrm{CO}_{2}$ emissions of the DES for each hour in the representative days. $O P E_{r, h}$ can be quantified using Equation 7, which uses EGEF to quantify the operating $\mathrm{CO}_{2}$ emissions from the grid.

$$
O P E_{r, h}=\mathrm{EGEF} \times \mathrm{OP}_{\text {grid }, r, h}
$$

\section{Two-Stage Stochastic Programming}

In this study, two-stage SP is used to optimize the sizing of the energy components considering uncertainties in the electricity demand, solar irradiance, wind speed, and EGEF in the DES.

Six steps are taken to perform two-stage SP. The diagram of these six steps is shown in Figure 4.

1. The decision variables in the first stage are solar PV arrays area, swept area (representing the capacity of wind turbines),
TABLE 2: Three different electricity purchasing cases considered in this study

\begin{tabular}{cccc}
\hline Name & Electricity from renewables & Electricity from the grid & EGEF \\
\hline Case 1 & $0 \%$ & $100 \%$ & 1 \\
\hline Case 2 & $71 \%$ & $29 \%$ & 0.29 \\
\hline Case 3 & $100 \%$ & $0 \%$ & 0 \\
\hline
\end{tabular}

and batteries' capacity. The sizing of these three energy components is initialized in the first stage.

2. The multi-objective optimization of the sizing of the energy components is performed in the first stage. An evolutionary algorithm, non-dominated sorting genetic algorithm II (NSGA-II) [45] is used by employing the Platypus library in Python [46] to perform the optimization in the first stage. The computation that is performed in the optimization process is time-consuming for two-stage SP. Distributing the computation among processing cores (i.e., parallelization) and parallel troubleshooting tools (like [47] and [48]) increase the performance of the optimization process.

3. Uncertainties in the four uncertain inputs (i.e., electricity demand, solar irradiance, wind speed, and electricity $\mathrm{CO}_{2}$ emissions) are revealed. This study uses the ten representative days to demonstrate the uncertainties in the four uncertain inputs.

4. The decision variables in the second stage are the hourly energy operation of the grid and batteries in each representative day. The operations of energy components are optimized for each of the ten representative days to minimize the operating cost while the electricity demand of the buildings is satisfied. The single-objective operation optimization of the energy components for each representative day is performed using the CPLEX solver in the Pyomo Python library ( [49]) to optimize the operation planning in the second stage.

5. The total cost (Equation 5) and operating $\mathrm{CO}_{2}$ emissions (Equation 6) are quantified for the initialized sizing of energy components in Step 1.

6. Steps $1-5$ are iterated until the optimum sizing to minimize the total cost and operating $\mathrm{CO}_{2}$ emissions are achieved using the NSGA-II algorithm, and a Pareto-front is generated.

\section{RESULTS AND DISCUSSION}

In this section, the results from the two-stage SP optimization of the sizing of energy components to minimize the total cost and operating $\mathrm{CO}_{2}$ emissions considering uncertainties are presented.

Three scenarios of purchasing electricity are considered, shown in Table 2, where values in the table are averaged annually. These three cases consider scenarios from purchasing electricity entirely from the grid to purchasing electricity entirely from renewable sources. 


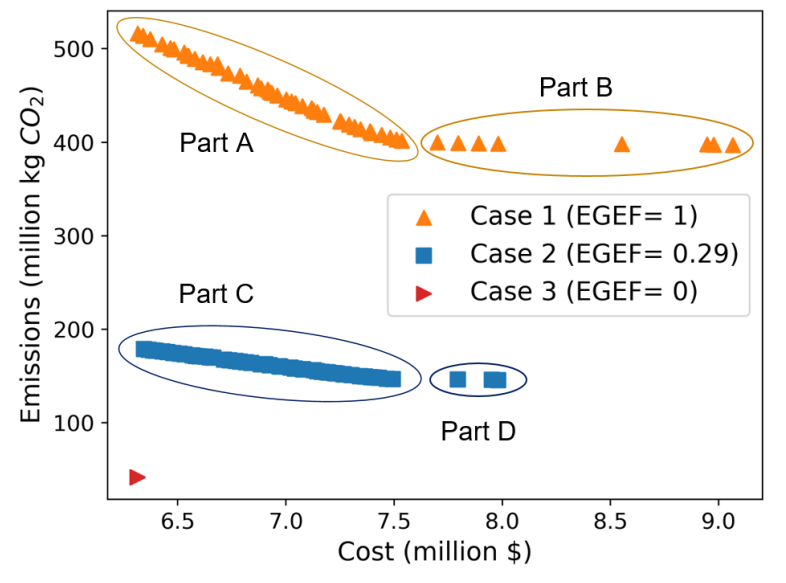

FIGURE 5: Cost and $\mathrm{CO}_{2}$ emissions trade-off for three electricity purchasing cases

In Figure 5, the trade-off between the total cost and operating $\mathrm{CO}_{2}$ emissions is shown for the three cases when 50 scenarios are generated for each case. Each scenario represents a different combination of sizing of solar PV systems, wind turbines, and batteries. For Case 1 and Case 2, the 50 generated scenarios can give decision-makers different combinations of cost and emissions trade-offs associated with different sizing of the three energy components. For Case 3, the Pareto-front consists of one scenario, shown as a single point in the figure.

In Figure 5, the cost of scenarios in Case 1 is from $\$ 6.3$ to $\$ 9.1$ million. The $\mathrm{CO}_{2}$ emissions of scenarios are from 397 to 516 million $\mathrm{kg}$. In Case 1, scenarios in Part $\mathrm{A}$, where the cost of forty-two scenarios increases from $\$ 6.3$ to $\$ 7.5$ million, their $\mathrm{CO}_{2}$ emissions decrease from 516.5 to 402.8 million $\mathrm{kg}$. But for scenarios beyond $\$ 7.5$ million, which are named Part B in the figure, the additional investment does not result in the same emission reduction.

In Figure 5, the cost of scenarios in Case 2 is from $\$ 6.3$ million to $\$ 8.0$ million. The $\mathrm{CO}_{2}$ emissions are from 146 to 179 million $\mathrm{kg}$. The $\mathrm{CO}_{2}$ emissions of scenarios are from 397 to 516 million kg. In Case 2, scenarios in Part C, where the cost of forty-seven scenarios increases from $\$ 6.3$ to $\$ 7.5$ million, their $\mathrm{CO}_{2}$ emissions decrease from 178.7 to 146.6 million $\mathrm{kg}$. But for scenarios beyond \$7.5 million, which are named Part D in the figure, the additional investment does not result in the same emission reduction.

In Figure 5, an additional cost investment in Part A and Part C scenarios results in a higher reduction in $\mathrm{CO}_{2}$ emissions compared to the same cost investment in Part B and Part D scenarios. Therefore, Part A and Part C scenarios are more likely to be selected compared to Part B and Part D scenarios.

The range of emissions due to using different combinations

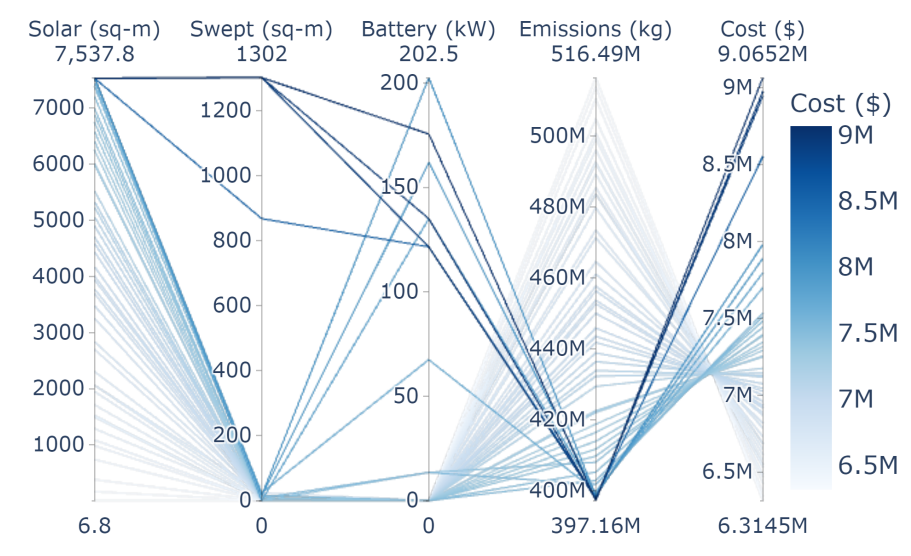

FIGURE 6: Parallel coordinates plot showing the variation in the optimum energy configurations to minimize the total cost and operating $\mathrm{CO}_{2}$ emissions for Case $1(\mathrm{EGEF}=1)$

of on-site renewables and batteries is 119 million $\mathrm{kg}$ in Case 1 and 33 million $\mathrm{kg} \mathrm{CO}$ in Case 2. The average emission reduction from Case 1 to Case 2 is approximately 294 million $\mathrm{kg} \mathrm{CO}_{2}$, and from Case 1 to Case 3 is approximately 414 million $\mathrm{kg} \mathrm{CO}$. These values indicate that the emission reduction from Case 1 to Case 2/case 3 is higher than the maximum emission reduction in each case. Therefore, using electricity purchasing cases (i.e., Case $1-3$ ), which are purchasing off-site renewable sources, results in higher emission reduction compared to using on-site renewables in this case study.

Figure 6 and Figure 7 show the optimum sizing of solar PV, wind turbines, and batteries for 50 scenarios to minimize the total cost and operating $\mathrm{CO}_{2}$ emissions for Case 1 and Case 2, respectively. Vertical coordinates in these figures show the range of solar PV array area $\left(\mathrm{m}^{2}\right)$ of the PV system, swept area $\left(\mathrm{m}^{2}\right)$ that represent the sizing of the wind turbines, the capacity of batteries $(\mathrm{kW})$, and their related total cost and emissions. Both figures show that in scenarios with larger solar array areas and swept areas (i.e., using more on-site renewable sources), the emissions are lower, and the cost is higher compared to the average cost and emissions over all of the scenarios in each case.

In Figure 6, the range of solar area of the PV system is from 6.8 to $7538.8 \mathrm{~m}^{2}$ (the maximum available rooftop area of the four buildings to install the solar PV systems). All of the scenarios have used a solar PV system. Higher values of the solar array area are related to higher cost and lower emissions, as expected. This figure shows that the easiest way to reduce emissions in Case 1 is by using more solar PV.

In Figure 6, thirty-one scenarios do not use wind turbines (0 swept area). A wind turbine is mostly selected in scenarios related to high cost and low emissions. The total cost of the ten 


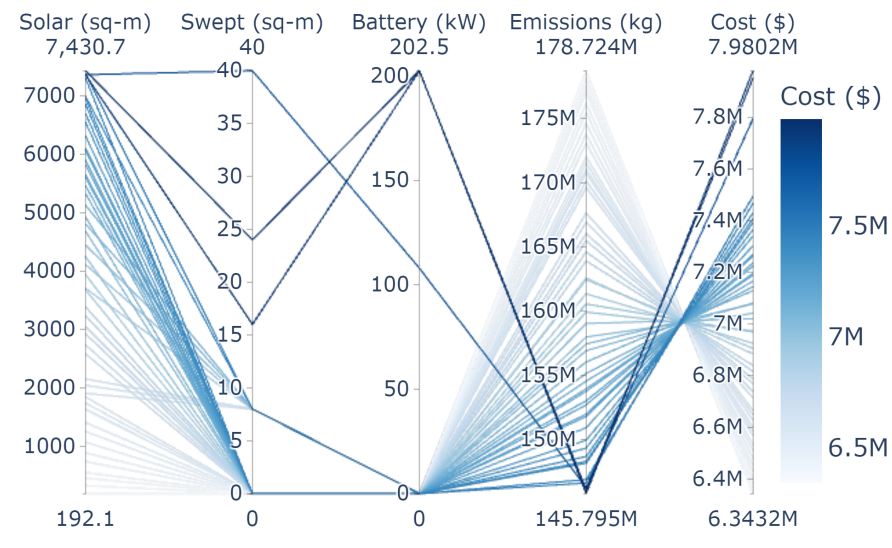

FIGURE 7: Parallel coordinates plot showing the variation in the optimum energy configurations to minimize the total cost and operating $\mathrm{CO}_{2}$ emissions for Case $2(\mathrm{EGEF}=0.29)$

scenarios with a swept area of less than $100 \mathrm{~m}^{2}$ is lower than $\$ 7.5$ million. These ten scenarios belong to Part A in Figure 5. The nine scenarios' total cost with the swept area of higher than 100 $\mathrm{m}^{2}$ is from $\$ 7.5$ to $\$ 9$ million. These nine scenarios belong to Part $B$ in Figure 5.

We discussed earlier that Part A scenarios are more likely to be selected than Part B scenarios. Therefore, scenarios with low swept areas (values less than $100 \mathrm{~m}^{2}$ ) are more likely to be selected than scenarios with high swept areas (values more than $100 \mathrm{~m}^{2}$ ) in Case 1.

The wind speed is low in the case study's location, where the average value is $1.5 \mathrm{~m} / \mathrm{s}$ in 2019 , using the actual data from NSRDB [20]. The cut-in speed, the speed when the wind turbine starts generating power, is from 2.6 to $4 \mathrm{~m} / \mathrm{s}$ on average [50]. Due to the present difference between cut-in speed and wind speed in the case study's location, the swept area of zero (not using wind turbines in the DES) is used in most scenarios in Case 1.

Forty scenarios do not use batteries ( 0 battery capacity). The ten scenarios that use batteries use large solar PV array areas and wind turbines. Also, the cost of these ten scenarios is higher than $\$ 7.4$ million, and they belong to Part B in Figure 5. Thus, it is unlikely that a decision-maker would select these scenarios.

In Figure 7, the range of solar area of the PV system is from $192.1 \mathrm{~m}^{2}$ to $7430.7 \mathrm{~m}^{2}$. All of the scenarios have used a solar PV system. Higher values of the solar array area are related to higher cost and lower emissions, as expected. This figure shows that the easiest way to reduce emissions in Case 2, similar to Case 1, is by using more solar PV.

In Figure 7, thirty-seven scenarios do not use wind turbines ( 0 swept area). A wind turbine is mostly selected in scenarios related to high cost and low emissions. The total cost of the remaining thirteen scenarios is from $\$ 6.6$ to $\$ 8.0$ million, and the scenarios mostly belong to Part C in Figure 5.

As discussed earlier, there is a difference between the cut-in speed (i.e., 2.6 to $4 \mathrm{~m} / \mathrm{s}$ ) and the average wind speed in the case study's location (i.e., $1.5 \mathrm{~m} / \mathrm{s}$ ). Due to the present difference, the swept area of zero (not using wind turbines in the DES) is used in most scenarios in Case 2.

Forty-seven scenarios do not use batteries ( 0 battery capacity). The three scenarios that use batteries use large solar PV array areas and small wind turbines. The cost of these three scenarios is higher than \$7.7 million, and they belong to Part D in Figure 5. Therefore, it is less likely that a decision-maker select these scenarios.

The detailed results of the sizing of energy components, total cost, and operating $\mathrm{CO}_{2}$ emissions for all of the 50 scenarios in Case 1, 2, and 3, along with the open-source code, are available in a public repository [51].

\section{CONCLUSION}

In this paper, a framework is developed to perform twostage stochastic programming in a district energy system. In this framework, the sizing of energy components is optimized to minimize the total cost and operating $\mathrm{CO}_{2}$ emissions considering uncertainties. This study's results show the trade-off between cost and $\mathrm{CO}_{2}$ emissions when different energy configurations and capacities are used for three different electricity purchasing cases.

The results show that the University of Utah is better to purchase off-site renewables compared to installing on-site renewables to reduce $\mathrm{CO}_{2}$ emissions. If the University of Utah wants to install on-site renewables, using the solar photovoltaic system is the easiest way to reduce emissions. Wind turbines are selected in scenarios related to high cost and low emissions due to the low average wind speed in the case study's location. Batteries are selected in scenarios that high on-site renewables are present. Each electricity purchasing case demonstrates some scenarios that are more likely to be selected by a decision-maker. In these scenarios, a larger emission reduction can be achieved with the same increment in the cost compared to other scenarios.

Considering the case study's circumstances, the University of Utah, some scenarios are more plausible than other scenarios. Second, the current purchasing electricity case of the University of Utah is closer to using a mix of $29 \%$ grid electricity and $71 \%$ off-site renewable sources. This mix of electricity is due to a new 25-year solar energy contract, which is expected to deliver power to the University of Utah by mid-2022 [23]. Third, among the scenarios in this electricity purchasing case, scenarios with a cost of less than $\$ 7.5$ million are more likely to be selected. In these scenarios, larger emission reductions are achieved with the same increment in the cost compared to other scenarios. Fourth, at the University of Utah, the space to install wind turbines is inadequate. Therefore, using large solar photovoltaic array areas without wind 
turbines is the best possible option for this case study due to the available rooftop area for solar photovoltaic installation at the University of Utah.

This framework can help with the multi-objective optimization of campus-level decisions. The framework considers various energy components and the uncertainties in electricity demand, solar irradiance, wind speed, and electricity emissions. This open-source framework [51] provides the opportunity to edit the decision space. Thus, a user can change the case study's location and edit the characteristics (e.g., capacities, efficiencies, investment cost, or O\&M cost) of energy components according to their own needs.

\section{ACKNOWLEDGMENT}

We appreciate the assistance and support provided by the University of Utah Department of Facilities Management. Special thanks go to Christopher Benson, who gave us access to campus buildings data.

\section{REFERENCES}

[1] U.S. Environmental Protection Agency. Sources of Greenhouse Gas Emissions. https://www.epa.gov/ghgemissions/ sources-greenhouse-gas-emissions. Accessed: 2021-02-22.

[2] IPCC, 2014. "Intergovernmental panel on climate change fifth assessment report". World Meteorological Org.

[3] National Oceanic and Atmospheric Administration, 2019. Climate change impacts. https: //www.noaa.gov/education/resource-collections/ climate-education-resources/climate-change-impacts. Accessed: 2020-01-07.

[4] United Nations Environment Programme, 2018. GOOD PRACTICE GUIDE District Energy. https://www.districtenergy.org/viewdocument/ good-practice-guide-district-energy. Accessed: 2020-1221.

[5] U.S. Environmental Protection Agency. Distributed Generation of Electricity and its Environmental Impacts. https://www.epa.gov/energy/ distributed-generation-electricity-and-its-environmental-impacts. Accessed: 2021-02-22.

[6] Aghamolaei, R., Shamsi, M. H., Tahsildoost, M., and O’Donnell, J., 2018. "Review of district-scale energy performance analysis: Outlooks towards holistic urban frameworks". Sustainable cities and society, 41, pp. 252-264.

[7] Center, B. P., 2021. "Annual energy outlook 2021". Energy Information Administration, Washington, DC.

[8] Kate Whitbeck, communications and relationship manager, Sustainability Office. Carbon Neutral BY 2050: Confronting Climate Change. https://sustainability.utah.edu/ commitments/. Accessed: 2021-02-24.
[9] Shapiro, A., and Philpott, A., 2007. "A tutorial on stochastic programming". Manuscript. Available at www2. isye. gatech. edu/ashapiro/publications. html, 17.

[10] Mavromatidis, G., Orehounig, K., and Carmeliet, J., 2018. "Design of distributed energy systems under uncertainty: A two-stage stochastic programming approach". Applied energy, 222, pp. 932-950.

[11] Al-Qahtani, K. Y., and Elkamel, A., 2011. Planning and integration of refinery and petrochemical operations. John Wiley \& Sons.

[12] Mavromatidis, G., Orehounig, K., and Carmeliet, J., 2018. "Uncertainty and global sensitivity analysis for the optimal design of distributed energy systems". Applied Energy, 214, pp. 219-238.

[13] Mavromatidis, G., Orehounig, K., and Carmeliet, J., 2018. "Comparison of alternative decision-making criteria in a twostage stochastic program for the design of distributed energy systems under uncertainty". Energy, 156, pp. 709-724.

[14] Mavromatidis, G., Orehounig, K., and Carmeliet, J., 2018. "A review of uncertainty characterisation approaches for the optimal design of distributed energy systems". Renewable and Sustainable Energy Reviews, 88, pp. 258-277.

[15] Wang, M., Yu, H., Jing, R., Liu, H., Chen, P., and Li, C., 2020. "Combined multi-objective optimization and robustness analysis framework for building integrated energy system under uncertainty". Energy Conversion and Management, 208, p. 112589.

[16] Wang, M., Yu, H., Lin, X., Jing, R., He, F., and Li, C., 2020. "Comparing stochastic programming with posteriori approach for multi-objective optimization of distributed energy systems under uncertainty”. Energy, 210, p. 118571.

[17] Jing, R., Wang, M., Zhang, Z., Wang, X., Li, N., Shah, N., and Zhao, Y., 2019. "Distributed or centralized? designing district-level urban energy systems by a hierarchical approach considering demand uncertainties". Applied Energy, 252, p. 113424.

[18] Fowler, K. M., Colotelo, A. H., Downs, J. L., Ham, K. D., Henderson, J. W., Montgomery, S. A., Vernon, C. R., and Parker, S. A., 2015. Simplified processing method for meter data analysis. Tech. rep., Pacific Northwest National Lab.(PNNL), Richland, WA, U.S.).

[19] Miller III, A. C., and Rice, T. R., 1983. "Discrete approximations of probability distributions". Management science, 29(3), pp. 352-362.

[20] Sengupta, M., Xie, Y., Lopez, A., Habte, A., Maclaurin, G., and Shelby, J., 2018. "The national solar radiation data base (nsrdb)". Renewable and Sustainable Energy Reviews, 89.

[21] U.S. Environmental Protection Agency, 2019. Basic information of air emissions factors and quantification. https: //www.epa.gov/air-emissions-factors-and-quantification/ basic-information-air-emissions-factors-and-quantification\# About\%20Emissions\%20Factors. Accessed: 2021-03-10. 
[22] Ghaemi, Z., and Smith, A. D., 2021. "Analyzing variability and decomposing electricity emission factors for three U.S. states". Manuscript submitted for publication. https://doi. org/10.31224/osf.io/kstgn.

[23] University of Utah Communications, 2020. The u reaches $71 \%$ renewable energy. https://sustainability.utah.edu/tag/ renewable-energy/. Accessed: 2021-03-24.

[24] U.S. Energy Information Administration. Utah Electricity Profile 2019. https://www.eia.gov/electricity/state/utah/ index.php. Accessed: 2021-03-03.

[25] ElNozahy, M., Salama, M., and Seethapathy, R., 2013. "A probabilistic load modelling approach using clustering algorithms". In 2013 IEEE Power \& Energy Society General Meeting, IEEE, pp. 1-5.

[26] Gaitani, N., Lehmann, C., Santamouris, M., Mihalakakou, G., and Patargias, P., 2010. "Using principal component and cluster analysis in the heating evaluation of the school building sector". Applied Energy, 87(6), pp. 2079-2086.

[27] Gelman, A., and Pardoe, I., 2006. "Bayesian measures of explained variance and pooling in multilevel (hierarchical) models". Technometrics, 48(2), pp. 241-251.

[28] Park, H.-S., and Jun, C.-H., 2009. "A simple and fast algorithm for k-medoids clustering". Expert systems with applications, 36(2), pp. 3336-3341.

[29] Jie, Y., Yibo, S., et al., 2019. "The study for data mining of distribution network based on particle swarm optimization with clustering algorithm method". In 2019 4th International Conference on Power and Renewable Energy (ICPRE), IEEE, pp. 81-85.

[30] D'Silva, J., and Sharma, U., 2020. “Unsupervised automatic text summarization of konkani texts using k-means with elbow method". Int J Eng Res Technol, pp. 2380-2384.

[31] Ni, Q., Pan, Q., Du, H., Cao, C., and Zhai, Y., 2015. "A novel cluster head selection algorithm based on fuzzy clustering and particle swarm optimization". IEEE/ACM transactions on computational biology and bioinformatics, pp. 76-84.

[32] Yuan, C., and Yang, H., 2019. "Research on kvalue selection method of k-means clustering algorithm". J-Multidisciplinary Scientific Journal, 2(2), pp. 226-235.

[33] Marutho, D., Handaka, S. H., Wijaya, E., et al., 2018. "The determination of cluster number at k-mean using elbow method and purity evaluation on headline news". In 2018 International Seminar on Application for Technology of Information and Communication, IEEE, pp. 533-538.

[34] Sun, Y., Gu, L., Wu, C. J., and Augenbroe, G., 2014. "Exploring hvac system sizing under uncertainty". Energy and Buildings, 81, pp. 243-252.

[35] Fu, R., Feldman, D. J., and Margolis, R. M., 2018. U.S. solar photovoltaic system cost benchmark: Q1 2018. Tech. rep., NREL, Golden, CO, U.S.

[36] NREL, 2016. "Distributed generation renewable energy estimate of costs". Accessed: 2021-03-15.
[37] NREL, 2020. News release: NREL research points to strategies for recycling of solar panels. https://www.nrel.gov/news/press/2020/ nrel-research-points-to-recycling-solar-panels.html. Accessed: 2021-03-15.

[38] Wiser, R. H., and Bolinger, M., 2019. "2018 wind technologies market report".

[39] Stehly, T. J., and Beiter, P. C., 2020. 2018 cost of wind energy review. Tech. rep., NREL, Golden, CO, U.S.

[40] McLaren, J., Gagnon, P., Anderson, K., Elgqvist, E., Fu, R., and Remo, T., 2016. Battery energy storage market: Commercial scale, lithium-ion projects in the U.S. Tech. rep., NREL, Golden, CO, U.S.).

[41] Cole, W., and Frazier, A., 2020. Cost projections for utilityscale battery storage: 2020 update. Tech. rep., NREL, Golden, CO, U.S.

[42] Smith, K., Saxon, A., Keyser, M., Lundstrom, B., Cao, Z., and Roc, A., 2017. "Life prediction model for gridconnected li-ion battery energy storage system". In 2017 American Control Conference (ACC), pp. 4062-4068.

[43] University of Utah Sustainability and Energy Management, 2020. Fy 2021 utility rates and bill collection disbursement. https://facilities.utah.edu/ sustainability-and-energy-management/resources/. Accessed: 2021-02-16.

[44] Ghaemi, Z., and Smith, A. D., 2020. "A review on the quantification of life cycle greenhouse gas emissions at urban scale". Journal of Cleaner Production, 252, p. 119634.

[45] Deb, K., Pratap, A., Agarwal, S., and Meyarivan, T., 2002. "A fast and elitist multiobjective genetic algorithm: Nsga-ii". IEEE transactions on evolutionary computation, 6(2).

[46] D. Hadka, 2019. Platypus: Multiobjective optimization in python. https://platypus.readthedocs.io.

[47] Taheri, S., Briggs, I., Burtscher, M., and Gopalakrishnan, G., 2019. "Difftrace: Efficient whole-program trace analysis and diffing for debugging". In 2019 IEEE International Conference on Cluster Computing (CLUSTER), IEEE, pp. 1-12.

[48] Taheri, S., Devale, S., Gopalakrishnan, G., and Burtscher, M., 2017. "Parlot: Efficient whole-program call tracing for hpc applications". In Programming and Performance Visualization Tools. Springer, pp. 162-184.

[49] Hart, W. E., Watson, J.-P., and Woodruff, D. L., 2011. "Pyomo: modeling and solving mathematical programs in python". Mathematical Programming Computation, 3(3).

[50] Office of Energy Efficiency \& Renewable Energy, U.S. Department of Energy . How do wind turbines survive severe storms? https://www.energy.gov/eere/articles/ how-do-wind-turbines-survive-severe-storms.

[51] Z. Ghaemi, A.D. Smith, 2021. Two-stage-stochasticprogramming. https://github.com/zahraghh/Two_Stage_SP/ tree/IMECE. 